\title{
The Architecture of Inference from SMT to ETB
}

\author{
Natarajan Shankar \\ SRI International Computer Science Laboratory
}

\begin{abstract}
Modularity plays a central role in logical reasoning. We want to be able to reuse proofs, proof patterns, theories, and specialized reasoning procedures. Architectures that support modularity have been developed at all levels of inference: SAT solvers, theory solvers, combination solvers and rewriters, SMT solvers, simplifiers, rewriters, and tacticsbased interactive theorem provers. Prior work has mostly focused on fine-grained modular inference. However, with the availability of a diverse range of high-quality inference tools, it has become important to systematically integrate these big components into robust toolchains. At SRI, we have been developing a framework called the Evidential Tool Bus (ETB) as a distributed platform for the coarse-grained integration of inference components into flexible, scriptable workflows. The talk describes the architecture of ETB along with some motivating applications.
\end{abstract}

